\title{
Staphylococcus pseudintermedius, both commensal and pathogen
}

\author{
DOROTA CHROBAK-CHMIEL, ANNA GOLKE, KOUROU DEMBELE*, KATARZYNA ĆWIEK, \\ MAGDALENA KIZERWETTER-ŚWIDA, MAGDALENA RZEWUSKA, MARIAN BINEK
}

\author{
Division of Microbiology, Department of Preclinical Sciences, Faculty of Veterinary Medicine, \\ Warsaw University of Life Sciences, Ciszewskiego 8, 02-786 Warsaw \\ *Division of Small Animal Internal Diseases, Department of Small Animals with Clinic, Faculty of Veterinary Medicine, \\ Warsaw University of Life Sciences, Nowoursynowska 159, 02-776 Warsaw
}

\section{Chrobak-Chmiel D., Golke A., Dembele K., Ćwiek K., Kizerwetter-Świda M., Rzewuska M., Binek M. Staphylococcus pseudintermedius, both commensal and pathogen \\ Summary}

Staphylococcus pseudintermedius is considered to be a both commensal and opportunistic canine pathogen. The anal, perineal and nasal locations appear to be the main S. pseudintermedius colonization sites, from which bacteria are transmitted to other body sites, causing secondary infections. When the immune system is compromised because of an underlying condition, the skin becomes susceptible to infection. Thus, the host's condition seems to play a crucial role in the pathogenesis of $\mathrm{S}$. pseudintermedius infections. There are some predisposing factors, one of which is atopic dermatitis. The pathogenic effects of S. pseudintermedius are mediated by several virulence factors, for instance superantigens, which play an important role by causing dermatitis. The immune system has evolved many different mechanisms to recognize and deal with pathogens, but bacteria have also developed various strategies to evade them. In this review, we focus on early stages of the innate immune response with particular emphasis on the mechanisms of recognition of staphylococci and the action of antimicrobial peptides.

Keywords: Staphylococcus pseudintermedius, pathogenicity, virulence factors, innate immune response

This review outlines Staphylococcus pseudintermedius as a normal inhabitant of the skin and mucosa of clinically healthy dogs and, on the other hand, as one of the most frequent bacterial pathogens isolated from clinical specimens of canine origin. As part of normal microbiota of most dogs, this bacterium does not cause any disease unless the resistance of the host is lowered and the skin barrier altered by predisposing factors, such as atopic dermatitis, medical and surgical procedures or immunosuppressive disorders (4). Despite its clinical importance, there is only very limited knowledge about the pathogenesis of $S$. pseudintermedius infections. More data are needed on the distribution of known and putative virulence factors, including cell wall-anchored proteins in the S. pseudintermedius population. Furthermore, the prevalence of individual virulence factors in distinct clonal lineages needs to be determined for the purpose of establishing their role in the pathogenesis of $S$. pseudintermedius-related diseases.

\section{Staphylococcus pseudintermedius as commensal bacteria in dogs}

S. pseudintermedius is considered as part of the resident microbiota in the nostril, oropharynx, and perianal region in dogs and other animal species. Thus, this bacterium is regarded as the main source of coagulase-positive staphylococci in the canine mucous membranes. It has been shown that, together with other microorganisms, $S$. pseudintermedius is part of the skin microbiome in healthy dogs (42). According to the authors, this bacterium has been isolated from $37 \%$ to $92 \%$ of healthy dogs (92). It should be noted that healthy dogs have a very diverse skin microbiome, which consists of many different microorganisms, including Micrococcus spp., coagulase-negative staphylococci, mainly Staphylococcus epidermidis and S. xylosus, $\alpha$-hemolytic streptococci, Clostridium spp., Propionibacterium acnes, Acinetobacter spp. and various Gram-negative aerobes (42). However, changes 
in the composition of canine skin microbiome occur in dogs suffering from atopic dermatitis. A reduction in the diversity of organisms living on the skin and often higher numbers of certain types of bacteria, such as S. pseudintermedius, were observed in unhealthy skin. These changes are associated with disorders in the skin barrier, but it still remains unknown which comes first: the damaged barrier or the altered microbiome.

\section{The occurrence of $\boldsymbol{S}$. pseudintermedius in dogs and its natural habitat}

Since 2005, Staphylococcus pseudintermedius has been considered as part of the normal microbiota of the skin and mucous membranes of the upper respiratory tract, the lower urogenital tract as well as the digestive tract, and it can be isolated from the nares, mouth, pharynx, anus, groin and forehead of healthy dogs and cats $(1,35,50,84)$. Many different carriage stages have been described. Isolation of $S$. pseudintermedius from animals may be associated with contamination, colonization or infection. Many authors suggest that colonization, consisting in the establishment of the pathogen at the appropriate way of entry, is the first stage of microbial infection (18). In other publications, different definitions of colonization and infection have been outlined. According to these authors, colonization is the presence and multiplication of bacteria at one or more body sites without visible clinical signs or immune reaction. Such bacteria may be present as the natural microbiota, environmental or endogenous factors. Infection, on the other hand, is regarded as an effect of bacterial invasion when microorganisms are multiplying in tissues and causing clinical manifestations of disease. However, there is much debate as to when colonization stops and infection begins. Contamination is the presence of bacteria on the skin and mucous membranes, but they can be easily eliminated (82). Hartman et al. (37) defined three types of skin colonization by $S$. pseudintermedius in dogs. Transient colonization was defined as the isolation of $S$. pseudintermedius only once during a sampling period of 12 months or more, while intermittent colonization was defined as the isolation of $S$. pseudintermedius at least twice and not necessarily consecutively during a sampling period of 12 months or more. In persistently colonized dogs, $S$. pseudintermedius was isolated from either the oral cavity or cranial hair coat twice or more in succession during a 12-month sampling period. Moreover, persistently colonized dogs have a greater load of $S$. pseudintermedius at colonization sites compared to transiently colonized animals. This may play a role in the development of diseases caused by $S$. pseudintermedius. Persistent carriage values among colonized dogs are higher than $50 \%$ and are higher than those for humans persistently colonized by $S$. aureus, which are estimated at about $20 \%$ (4). Moreover, the anal, perineal and nasal loca- tions appear to be the main colonization sites (25). There is a suggestion that the anus and nares act as the ecological niche for S. pseudintermedius, and from here the bacterium can be transmitted to other body sites, such as the hair shafts, sites of infection, other dogs in the household, or even humans having regular contact with the dog (19).

\section{Factors predisposing to Staphylococcus pseudintermedius colonization in dogs}

It is known that there are some factors predisposing dogs to higher carriage rates of $S$. pseudintermedius. One of such factors is canine atopic dermatitis (AD), which is a chronic inflammatory skin disease associated with environmental allergies (24). The literature data indicate that several breeds seems to be at higher risk for $\mathrm{AD}$ and thus at higher risk for colonization and secondary infections caused by $S$. pseudintermedius, for instance: Boxer, Bull Terrier, Chow Chow, Chinese Shar-Pei, Cocker Spaniel, French Bulldog, Fox Terrier, German Shepherd Dog, Golden Retriever, Irish Setter, Labrador Retriever, Poodle, Dalmatian, West Highland White Terrier, etc (48). Skin lesions in atopic dogs are often associated with chronic pruritus or self-trauma and include alopecia, crusting, lichenification and hyperpigmentation, which may lead to superficial staphylococcal pyoderma, Malassezia spp. infection and bacterial overgrowth (48). The etiology and pathogenesis of canine AD is complex and involves genetic predisposition, weakened skin barrier function, and immunological disorders. Dogs suffering from $\mathrm{AD}$ are probably genetically predisposed to become sensitized to environmental allergens, such as home dust, grass, tree and weed pollens, mold spores, epidermal antigens, insect antigens, etc (59). Thus, higher carriage rates of $S$. pseudintermedius were observed in dogs with atopic dermatitis (87\%) than in healthy individuals (37\%) (27). Interestingly, it was described that $S$. pseudintermedius strains adhere better to corneocytes collected from atopic dogs or dogs belonging to specific breeds, such as Boxers and Bull Terriers (30, 65). This may be explained by changes in skin receptors specific for staphylococci, greater expression of intercellular adhesion, and a different distribution pattern of fibronectin in atopic skin $(11,78,94,96)$. Due to the fact that staphylococcal adherence to corneocytes is considered to be a crucial step for skin colonization and subsequent infection, several research studies on this aspect of $S$. pseudintermedius have already been conducted and published $(56,66,78,96)$. Bacterial adherence to the skin involves interaction between adhesins on the bacterial surface and receptors on various skin components, including the keratinocytes, corneocytes and extracellular matrix proteins of the skin (97). The studies show that adherence to these components is mediated by specific receptor-ligand interactions. $S$. pseudintermedius binds to proteins 
such as fibrinogen, fibronectin, cytokeratin and elastin, functioning as ligands (91). If the bacterium is unable to adhere, it will be removed from the host by natural processes such as desquamation of the skin and grooming (97). Corneocytes are completely flattened cells, with neither nucleus nor cytoplasmic organelles. These cells are therefore dead, but remain active: the result of the final phase of keratinocyte differentiation, they are filled with keratin and other products, such as lipids, fatty acids and ceramides (69). A study conducted by McEwan et al. (66) proved that $S$. pseudintermedius adheres to both lesional and non-lesional canine atopic skin. However, stronger adherence was observed to corneocytes collected from skin lesions in atopic dogs compared to those collected from non-lesional areas, suggesting that ligands for bacterial surface proteins were expressed at higher levels. This may be important in the colonization of atopic skin by this microorganism. The aforementioned authors suggest that a T-helper type 2 (Th2)-mediated inflammation in canine atopic skin facilitates binding by S. pseudintermedius, which leads to the colonization of the skin and predisposes to staphylococcal pyoderma observed in canine atopic dermatitis. Additionally, it seems likely that the higher prevalence of staphylococcal colonization in atopic dogs is behind the high frequency of recurrent skin infections observed in these dogs.

Paul et al. (78) have studied the adherence of commensal $S$. pseudintermedius strains to canine corneocytes. Bacteria and corneocytes were collected from healthy dogs with different colonization statuses: persistently colonized, intermittent and non-carriers. Furthermore, all staphylococcal strains showed different properties in PFGE and MLST analyses. Results obtained in this study showed that, in general, all staphylococcal strains adhered better to corneocytes from persistent carriers. However, the authors also noted that one strain isolated from a persistently colonized dog adhered strongly to corneocytes collected from an intermittent carrier. In addition, they pointed out that the strain and the corneocytes had been obtained from different animals. This may indicate that preferential binding is not influenced by strain adaptation to individual hosts. Therefore, the authors suggested that the adherence of $S$. pseudintermedius to canine corneocytes is mainly related to host factors and only marginally influenced by strain factors. In line with that, Schmidt et al. (91) compared the ability of S. pseudintermedius strains isolated from healthy and atopic dogs to adhere to immobilized human fibronectin and epidermal cytokeratin and canine fibrinogen in vitro. They found that most staphylococcal strains adhered strongly, and they did not observe any significant difference between isolates from atopic and healthy dogs or from lesional and non-lesional areas on the skin of atopic dogs. Those results also confirm that $S$. pseudintermedius is equipped with specific microbial components recognizing adhesive matrix molecules that bind to host proteins. Other studies have clarified that among $S$. pseudintermedius there was no correlation between virulence and adherence to canine corneocytes in vitro, since no significant difference has been found between $S$. pseudintermedius strains isolated from pyoderma lesions and strains isolated from healthy dogs (87). Research concerning bacterial adherence to corneocytes is of high importance and may reveal potential options for the prophylaxis and treatment of canine staphylococcal infections.

\section{Staphylococcus pseudintermedius as pathogenic bacteria in dogs}

In addition to being commensal on mucous membranes and skin, S. pseudintermedius is also an opportunistic pathogen causing pyogenic infections in animals. This species is mainly adapted to the family of Canidae, such as dogs and foxes (4). However, according to the literature, it has also been isolated from cats, horses, donkeys, cattle, birds, rats and rarely from humans $(41,43,85,110)$. Canine infections caused by S. pseudintermedius are mostly skin and ear infections, endometritis, cystitis, post-operative wound infections, urinary tract infections and respiratory tract infections $(12,20,70,71,86,93,111)$.

\section{Pathogenicity and the prevalence of S. pseudintermedius in diseased dogs}

As already mentioned, it has been proved that $S$. pseudintermedius strains isolated from pyoderma lesions and from healthy skin do not differ in their ability to adhere to corneocytes isolated from healthy dogs. In line with this, it was found that strains isolated from pyodermal lesions were as genetically heterogeneous as strains isolated from healthy dogs (27). On the other hand, it has been described that $S$. pseudintermedius obtained from diseased dogs (pyoderma patients) shows a significantly greater adherence to keratinocytes from atopic skin and pyoderma lesions than to keratinocytes from healthy skin (64). Some studies suggest that the development of secondary skin infections in atopic dogs caused by $S$. pseudintermedius is mainly related to host factors, such as the exposition and availability of a greater number of ligands on host keratinocytes that enable a greater number of bacteria to adhere to the skin (64). In general, $S$. pseudintermedius is a causative agent of secondary infections when the skin or mucous membranes are compromised in some way. A minor trauma or immunosuppression may predispose to the development of such infections. Moreover, staphylococcal skin infections may also be associated with other factors, for instance, with intercurrent parasitic (Demodex canis, scabies or fleas) or fungal infections, allergic conditions, endocrinopathies (hypothyroidism, Cushing's disease), follicular 
dysplasia disorders or keratinization disorders (29). Furthermore, a higher load of $S$. pseudintermedius in persistently colonized dogs increases the risk for the development of disease. When the host becomes immunocompromised, staphylococci more easily overcome the host's immunological defences. Moreover, due to the high load of $S$. pseudintermedius at certain body sites (perineum, nasal and oral cavities) in persistent carriers, the chances of transmission of these bacteria to other sites on the canine body are increased (37).

\section{Staphylococcus pseudintermedius as the major causative agent of canine pyoderma}

Pyoderma is one of the most common skin diseases in dogs. A number of different clinical manifestations of canine pyoderma are recognized, depending on lesion type and distribution. Classification by lesion depth is considered useful because the choice of antimicrobial therapy may vary according to the cutaneous tissue layers affected $(45,72,100)$. Bacterial pyodermas are either simple infections or complex infections. Simple infections are those occurring in young animals and are triggered by one-time or simple events, for instance, flea infestation. Complex infections are recurrent and are associated with underlying diseases. Both simple and complex infections can be superficial or deep. Bacterial pyodermas limited to the epidermis and hair follicles are referred to as surface or superficial pyodermas, whereas those that involve the dermis or cause furunculosis are referred to as deep pyodermas. Etiologic classification refers to the pathogenic organism involved in the infection (eg, staphylococci, streptococci, etc) (100). In the course of the disease various clinical signs are observed, including pustules, crusted papules and erythema, as well as impetigo, furunculosis and abscesses (102). In dogs, superficial bacterial folliculitis (SBF) is the commonest form of canine pyoderma (40). Superficial pyoderma is defined as a superficial bacterial infection of the epidermis and hair follicles, and is usually secondary to allergic, parasitic, endocrine, immune-mediated, conformational or keratinization (seborrheic) disorders. There are some predisposing factors for canine pyoderma, for instance: hair follicles lack a lipid plug; the canine stratum corneum is far thinner and more compact than that of any other species; dogs have a high skin $\mathrm{pH}$ and decreased cutaneous defensins in cases of pyoderma secondary to atopic dermatitis (58, 62). S. pseudintermedius can easily colonize inflamed seborrhoeic skin. In fact, in inflamed skin, epidermal proliferation and desquamation are increased, and the skin is more humid and warmer. These alterations of the skin surface micro-environment promote the multiplication of bacteria. Furthermore, self-trauma by scratching, rubbing, chewing or licking hair and skin due to pruritus further degrade epidermal defences and favour inoculation of bacteria into the skin as well as the leakage of serum, which is a source of nutrients for bacteria (60). In addition, there is evidence from an experimental model of canine cutaneous type I hypersensitivity that intradermal injection of a mast cell degranulator or histamine renders the overlying epidermis more permeable to bacterial antigens (61). Thus, allergic skin disease is probably one of the most common factors predisposing to canine pyoderma. In the case of skin diseases, including canine pyoderma, bacteria present on the skin surface, capable of producing a wide range of virulence factors, seek a window of opportunity for starting the proliferation and invasion of the host organism.

\section{Virulence factors and their role in staphylococcal pathogenicity}

S. pseudintermedius has the capacity to produce a number of virulence factors, including some which are functionally related to virulence factors of $S$. aureus. These virulence factors are involved in almost all processes from colonization of the host to bacterial nutrition and dissemination (29). Briefly, virulence factors produced by $S$. pseudintermedius can be divided into the following groups: cell-associated components, exoenzymes and exotoxins. Staphylococcal cell-wall-anchored (CWA) proteins, such as microbial surface components recognizing adhesive matrix molecules (MSCRAMMs), play an important role in bacterial attachment to the host extracellular matrix. In $S$. pseudintermedius, surface proteins such as staphylococcal cell wall proteins (SpsA-R), which bind to fibronectin and fibrinogen, facilitate bacterial attachment to tissues. Geoghegan et al. (34) discovered that $S$. pseudintermedius has the capacity to bind to fibrinogen, fibronectin and cytokeratin 10, indicating the presence of surface adhesins, which could explain how $S$. pseudintermedius adheres to canine corneocytes during colonization and infection. In $S$. pseudintermedius, two cell wall-associated proteins, SpsD and SpsL, were described as playing a fundamental role in host tissue colonization and the pathogenesis of canine skin diseases (79). In addition, IgG specific for SpsD and SpsL occur in dogs with pyoderma, which leads to the conclusion that this protein is expressed during infection (4). Interestingly, strains isolated from pyodermal lesions have been shown to produce a mucoid substance known as slime that probably supports the adherence (49). In 2009, Moodley and co-workers reported that $S$. pseudintermedius produces an immunoglobulin-binding protein similar to staphylococcal protein A produced by $S$. aureus (70). Protein A, as well as other structural features, including capsular polysaccharide and teichoic acids, interfere with opsonization and subsequent phagocytosis. Protein A binds IgG molecules by their $\mathrm{Fc}$ region, and thus in serum bacteria bind $\mathrm{IgG}$ molecules in the wrong orientation, which leads to the inhibition of phagocytosis (31). 
Regarding the exoenzymes, it is worth mentioning free coagulase. The production of free coagulase by S. pseudintermedius also protects these bacteria from phagocytic and immune defences by causing localised clotting (31). Coagulase is an extracellular protein that binds to prothrombin in the host to form a complex called staphylothrombin. Protease activity is activated in the complex, which leads to the conversion of fibrinogen to fibrin.

Some of the exotoxins produced by S. pseudintermedius are known as superantigens (SAgs). Toxins that have superantigenic activity stimulate $T$ cells nonspecifically without normal antigenic recognition (39). Such stimulation of the T lymphocytes in an uncontrolled manner results in fever, shock and death (81). SAgs directly cross-link conserved regions of the variable domains, the V $\beta$-element of T cell receptors (TCRs) with major histocompatibility complex (MHC) class II molecules on antigen-presenting cells. This results in a strong stimulation of $\mathrm{T}$ cells, which respond with proliferation and massive cytokine release. These interactions between superantigens, TCRs and MHC class II molecules play a role in the induction or progression of dermatitis, whereas such a large amount of cytokines can either cause or enhance the ongoing inflammatory process (31). Some strains of S. pseudintermedius produce enterotoxins and toxic shock syndrome toxin-1 (TSST-1), which exhibit superantigenic activity. There are several reports on S. pseudintermedius strains isolated from dogs producing staphylococcal enterotoxins (SEs), such as SEA, SEB, SEC and SED. In addition, this microorganism is also able to produce two unique enterotoxins: SE-int and $\mathrm{SEC}_{\text {canine, }}$ which is a $\mathrm{SEC}$ variant (102). The frequent occurrence of strains producing $\mathrm{SEC}_{\text {canine }}$ in pyoderma lesions indicates that this toxin, in combination with exfoliative toxins, may play a role in the development of pyoderma. In dogs, there is increasing evidence suggesting that some lesions of superficial bacterial skin infections are caused by $S$. pseudintermedius producing exfoliative toxins (5). Staphylococcal exfoliative toxins are known to digest desmoglein (Dsg) 1, a desmosomal cell-cell adhesion molecule, thus causing intraepidermal splitting in human bullous impetigo, staphylococcal scalded skin syndrome and swine exudative epidermitis ("greasy pig disease") (47). It was proved that Staphylococcus intermedius exfoliative toxin (SIET), which is produced by $S$. pseudintermedius strains, has a rounding effect in cultured epithelial cells and an exfoliative effect in 1-day-old chickens, hamsters and dogs, but not in rats or mice (104). Dogs injected with purified SIET developed lesions with positive Nikolsky's sign (exfoliation of skin when rubbed) with erythema and crusting which resemble those of canine pyoderma (104). On the other hand, other studies revealed that intradermal injection of recombinant SIET in dogs did not cause any lesions, either clinically or histopatho- logically (47). Thus, the biological significance of this toxin remains unclear $(47,104,113)$. Futagawa-Saito et al. (32) described a novel exfoliative toxin (EXI) produced by $S$. pseudintermedius. However, studies on the prevalence of the EXI gene, encoding for this toxin in $S$. pseudintermedius, revealed that it was not present in all strains tested. This gene was identified in $23 \%$ of $43 \mathrm{~S}$. pseudintermedius strains collected from dogs with pyoderma (32). Additionally, in other studies, EXI-negative strains were isolated from dogs with diagnosed bullous impetigo. Moreover, a new gene, encoding another protein that resembled EXI, was found. The authors proposed the renaming of EXI as EXPA (exfoliatin of $S$. pseudintermedius type A), and the novel protein was named EXPB (exfoliatin of S. pseudintermedius type B). Moreover, it was shown that both EXPA and EXPB could digest canine desmoglein-1 (Dsg-1) and caused subcorneal splits in the epidermis when injected in mice, which resembles mechanisms involved in the pathogenesis of intraepidermal splitting in canine impetigo $(46,47)$.

$S$. pseudintermedius also produces several toxins, such as hemolysins and leukocidin, that damage the membranes of host cells. Alpha-toxin is a pore-forming toxin which may have a role in escape from the phagosome, while $\beta$-toxin has sphingomyelinase activity with a high affinity for sphingomyelin. The $\gamma$-toxin (also called leukotoxin) and leukocidin (Luk-I) are proteins that act together to destroy leukocytes and lipid membranes and appear to be crucial virulence factors in necrotizing lesions. Leukocidin consists of two distinct, synergistically interacting proteins designated as Luk-S and Luk-F, which belong to two different classes. Together, these proteins form pores in the membrane of polymorphonuclear cells, thus causing a cytolytic effect on such cells (80). Because of their dermonecrotic activity, leukotoxin and leukocidin of $S$. pseudintermedius can be potentially involved in the development of skin diseases in dogs.

\section{Innate immune response against staphylococcal skin infections}

Current knowledge about the innate immune response against $S$. preudintermedius is limited. However, some speculations can be made based on the data concerning the key elements of this response against other staphylococci, such as $S$. aureus. Moreover, it is important to note that a significant part of our knowledge of the immune response within the skin concerns mainly infections, not carriage. The innate immune system is the first line of defence against invading pathogens. It produces a rapid response, which may be crucial for eliminating the infectious agent. However, the pathogen must first penetrate physical barriers, such as the stratum corneum. If staphylococci succeed in binding to the host surface components, such as fibronectin, fibrinogen and cytokeratins derived from epidermal 
keratinocytes, they will encounter innate immune response proteins, such as the complement components and transferrin, which are present in the stratum corneum $(10,33,105)$. However, $S$. aureus synthesizes on its surface transferrin-binding proteins and capsular polysaccharides that inhibit $\mathrm{C} 3 \mathrm{~b}$ or antibody recognition and interfere with opsonisation $(16,103)$.

More sophisticated mechanisms of eliminating infectious agents strictly depend on the recognition of pathogen-associated molecular patterns (PAMPs) (28). In the case of bacterial infections, toll-like receptors (TLRs) and nucleotide-binding, oligomerization domain (NOD)-like receptors (NLRs) are the most important classes of innate pattern recognition receptors (PRRs) present on the cell surface or inside the host's immune and non-immune cells. When activated, TLRs and NLRs further trigger the activation of the transcription factor NF- $\kappa \mathrm{B}$ (nuclear factor kappa-lightchain-enhancer of activated B cells) or other transcription factors, which control the expression of genes encoding cytokines, chemokines and costimulatory molecules necessary for the activation of the defence response culminating in the phagocytosis of bacteria and recruitment of immune cells (109). Among all TLRs, TLR2 is essential for the recognition of staphylococcal components, and it is present on the surface of many different cell types in the skin, including keratinocytes (67). It may form heterodimers with TLR1 or TLR6, and each dimer will recognize different ligands. It is believed that TLR2/TLR1 dimer recognizes triacyl and TLR2/TLR6 diacyl staphylococcal lipopeptydes. In addition, Panton-Valentine toxin and Phenol Soluble Modulins (PSMs) are suspected to be potent TLR2 ligands $(7,22,36,38,99,101)$. The TLR2 ligand function of peptidoglycan (PGN) and lipoteichoic acid (LTA) remains controversial. It is considered that PGN is recognized by TLR 2 in cooperation with CD14 and CD36 or that it is rather recognized by NOD2, another innate immune receptor (106).

Other TLRs may be taken into account as potential receptors recognizing staphylococcal ligands. TLR4 seems to be activated by $S$. aureus leukocidin (44). Moreover, $S$. aureus may activate TLR9, which results in the production of type I interferons (IFNs) in dendritic cells, and as a result, similar signalling pathways are stimulated as in the case of viral infections $(68,77)$.

There is also another intracellular cytoplasmic receptor that can recognize staphylococci. NOD2, belonging to a family of PRRs known as NLRs, senses muramyl dipeptide, a peptidoglycan breakdown product, which leads to the production and secretion of cytokines, such as $\operatorname{IL}-1 \beta(23,54)$.

In the case of staphylococcal infections, other pathways of innate immunity mechanism activation are also possible. S. aureus protein A (SpA) can mimic tumor necrosis factor alpha (TNF- $\alpha)$ and directly activates
TNF receptor 1 (TNFR1), which elicits the release of cytokines (14). In kerationocytes, the adherence of $S$. aureus also causes the induction of TNF- $\alpha$ and TNFR1 expression $(3,55)$. Various virulence factors of staphylococci may stimulate the inflammatory response. It was shown that $S$. aureus alpha toxin induces the production of cytokines $(21,75)$, while $\beta$-hemolysin induces an inflammatory response by damaging monocytes, which contain mediators such as IL-1 $\beta$ (107).

Activation of TLRs and NOD2 in host cells will also result in the stimulation and release of antimicrobial peptides (AMPs), mainly defensins and cathelicidins, which have a direct bacteriostatic or bactericidal activity against $S$. aureus and $S$. pseudintermedius $(8,9)$. AMPs could stimulate the release of prostaglandin, induce recruitment of immune cells, enhance phagocytosis, promote angiogenesis and support wound healing. The character of these responses depends on the host, the type of infected cells and virulence factors of the bacterium $(8,9,63)$. In humans, $\beta$-defensin 2 (hBD2) and hBD3 are believed to be the most important in defence against staphylococci (52). In dogs, several $\beta$-defensins (cBD1, 2, 3, 101, 107) and one cathelidicin (cCath) are present in the skin, but the results concerning their role in staphylococcal infections and $\mathrm{AD}$ are contradictory $(17,57,73,89)$. It is difficult to speculate as to the cause of such differences, but there is no doubt that canine keratinocytes can produce AMPs both in vitro and in vivo $(57,89)$, and these peptides show effective antibacterial activity against $S$. pseudintermedius $(26,57,90)$.

Unfortunately, staphylococci are able to modulate their sensitivity to defensins and other AMPs by changing the composition and net charge of LTA or phospholipids, and some of these mechanisms are connected with methicillin resistance $(53,83)$. Comparison of MSSA with MRSA strains revealed that MRSA strains were less susceptible to AMPs (15). Indeed, numerous studies have shown a correlation between methicillin and human cathelicidin LL-37 resistance $(76,88,108)$. Moreover, staphylococcal metalloproteinase aureolysin can cleave LL-37, and iron-regulated surface protein A (isdA) can impart resistance to human $\beta$-defensin 2 $(13,95)$. Furthermore, staphylococcal superantigens, such as (SSL) proteins, are also capable of disrupting the host's innate immune response. Among these, SSL3 blocks TLR2 activation through direct extracellular interaction with this receptor, inhibiting TNF- $\alpha$ production $(6,51,98,112)$. Recently, a new virulence factor of $S$. aureus has been discovered. It was identified as a homologue of the human TIR-domain and was named staphylococcal TIR-domain protein (TirS), which appeared to interfere with signalling through TLR2 in kidney, macrophage and keratinocyte cell lines. In a systemic mouse infection model, TirS also enhanced bacterial accumulation in several organs (2). 
In addition, some host-related factors may also impede the recognition of pathogens by PRRs. Certain polymorphisms in human genes that encode TLRs may affect susceptibility to infection. Humans with TLR2 Arg753Gln polymorphism show increased susceptibility to infections with Gram-positive bacteria. Moreover, TLR2 and BD1 polymorphisms were connected with cases of severe AD (74).

In conclusion, it is clear that a large number of staphylococcal molecules interact with the innate immune system, and probably many more are still waiting to be discovered. It is well known that staphylococci can be recognized by many different receptors of innate immunity, and therefore can activate various signalling pathways, leading to proinflammatory cytokine release. Although a lot of research focused on peptidoglycan and LTA recognition by TLR2 and Nod2, much less is known about the impact of different groups of staphylococcal virulence factors on the inflammatory response. Moreover, other receptors, such as TLR9 and TNFR1, may also contribute to the activation of transcription factors and production of cytokines.

\section{References}

1.Abraham J. L., Morris D. O., Griffeth G. C., Shofer F. S., Rankin S. C.: Survivalence of healthy cats and cats with inflammatory skin disease for colonization of the skin by methicillin-resistant coagulase-positive staphylococci and Staphylococcus schleiferi subsp. schleiferi. Vet. Dermatol. 2007, $18,252-259$.

2.Askarian F., van Sorge N. M., Sangvik M., Beasley F. C., Henriksen J. R., Sollid J. U., van Strijp J. A., Nizet V., Johannessen M.: A Staphylococcus aureus TIR domain protein virulence factor blocks TLR2-mediated NF- $\mathrm{KB}$ signaling. J. Innate Immun. 2014, 6, 485-498.

3. Aufiero B., Guo M., Young C., Duanmu Z., Talwar H., Lee H. K., Murakawa G. J.: Staphylococcus aureus induces the expression of tumor necrosis factor-alpha in primary human keratinocytes. Int. J. Dermatol. 2007, 46, 687-694.

4. Bannoehr J., Guardabassi L.: Staphylococcus pseudintermedius in the dog: taxonomy, diagnostics, ecology, epidemiology and pathogenicity. Vet. Dermatol. 2012, 23, 253-266

5. Banovic F., Linder K., Olivry T.: Clinical, microscopic and microbial characterization of exfoliative superficial pyoderma-associated epidermal collarettes in dogs. Vet. Dermatol. 2016, 28, 107-e23.

6. Bardoel B. W., Vos R., Bouman T., Aerts P. C., Bestebroer J., Huizinga E. G., Brondijk T. H., van Strijp J. A., de Haas C. J.: Evasion of Toll-like receptor 2 activation by staphylococcal superantigen-like protein 3. J. Mol. Med. 2012, 90, 1109-1120

7. Blander J. M., Medzhitov R.: Regulation of phagosome maturation by signals from Toll-like receptors. Science 2004, 304, 1014-1018

8. Brogden K. A., Ackermann M., McCray Jr P. B., Tack B. F.: Antimicrobial peptides in animals and their role in host defences. Int. J. Antimicrob. Agents 2003, 22, 465-478.

9. Cederlund A., Gudmundsson G. H., Agerberth B.: Antimicrobial peptides important in innate immunity. FEBS J. 2011, 278, 3942-3951.

10. Chavakis T., Preissner K. T., Herrmann M.: The anti-inflammatory activities of Staphylococcus aureus. Trends Immunol. 2007, 28, 408-418.

11. Cho S. H., Strickland I., Boguniewicz M., Leung D. Y.: Fibronectin and fibrinogen contribute to the enhanced binding of Staphylococcus aureus to atopic skin. J. Allergy Clin. Immunol. 2001, 108, 269-274.

12. Chrobak D., Kizerwetter-Świda M., Rzewuska M., Moodley A., Guardabassi L., Binek M.: Molecular characterization of Staphylococcus pseudintermedius strains isolated from clinical samples of animal origin. Folia Microbiol. 2011, $56,415-422$

13. Clarke S. R., Mohamed R., Bian L., Routh A. F., Kokai-Kun J. F., Mond J. J., Tarkowski A., Foster S. J.: The Staphylococcus aureus surface protein IsdA mediates resistance to innate defenses of human skin. Cell Host Microbe. 2007, 1, 199-212.

14. Classen A., Kalali B. N., Schnopp C., Andres C., Aguilar-Pimentel J. A., Ring J., Ollert M., Mempel M.: TNF receptor I on human keratinocytes is a binding partner for staphylococcal protein A resulting in the activation of NF kappa B, AP-1, and downstream gene transcription. Exp. Dermatol. 2011, 20, 48-52.

15. Cole A. M., Liao H. I., Stuchlik O., Tilan J., Pohl J., Ganz T.: Cationic polypeptides are required for antibacterial activity of human airway fluid. J. Immunol. 2002, 169, 6985-6991.

16. Cunnion K. M., Hair P. S., Buescher E. S.: Cleavage of complement C3b to $\mathrm{iC} 3 \mathrm{~b}$ on the surface of Staphylococcus aureus is mediated by serum complement factor I. Infect. Immun. 2004, 72, 2858-2863.

17. Damme C. M. M. van, Willemse T., van Dijk A., Haagsman H. P., Veldhuizen E. J. A.: Altered cutaneous expression of $\beta$-defensins in dogs with atopic dermatitis. Mol. Immunol. 2009, 46, 2449-2455.

18. Dani A.: Colonization and infection. Cent. European J. Urol. 2014, 67, 86-87.

19. Devriese L. A., DePelsmaecker K.: The anal region as a main carrier site of Staphylococcus intermedius and Streptococcus canis in dogs. Vet. Record. 1987, 121, 302-303.

20. Diribe O., Thomas S., AbuOun M., Fitzpatrick N., La Ragione R.: Genetic relatedness and characterization of Staphylococcus pseudintermedius associated with post-operative surgical infections in dogs. J. Med. Microbiol. 2015, 64, 1074-1081.

21. Dragneva Y., Anuradha C. D., Valeva A., Hoffmann A., Bhakdi S., Husmann M.: Subcytocidal attack by staphylococcal alpha-toxin activates NF- $\kappa \mathrm{B}$ and induces interleukin-8 production. Infect. Immun. 2001, 69, 2630-2635.

22. Dziarski R., Gupta D.: Staphylococcus aureus peptidoglycan is a toll-like receptor 2 activator: a reevaluation. Infect. Immun. 2005, 73, 5212-5216.

23. Elinav E., Strowig T., Henao-Mejia J., Flavell R. A.: Regulation of the antimicrobial response by NLR proteins. Immunity 2011, 34, 665-679.

24. Favrot C., Steffan J., Seewald W., Picco F.: A prospective study on the clinical features of chronic canine atopic dermatitis and its diagnosis. Vet. Dermatol. 2010, 21, 23-30.

25. Fazakerley J., Nuttall T., Sales D., Schmidt V., Carter S. D., Hart C. A., McEwan N. A.: Staphylococcal colonization of mucosal and lesional skin sites in atopic and healthy dogs. Vet. Dermatol. 2009, 20, 179-184.

26. Fazakerley J. R., Crossley J., McEwan N., Carter S., Nuttall T.: In vitro antimicrobial efficacy of beta-defensin 3 against Staphylococcus pseudintermedius isolates from healthy and atopic canine skin. Vet. Dermatol. 2010b, 21, 463-468.

27. Fazakerley J. R., Williams N., Carter S., McEwan N., Nuttal T.: Heterogeneity of Staphylococcus pseudintermedius isolates from atopic and healthy dogs. Vet. Dermatol. 2010a, 21, 578-585.

28. Fedtke I., Gotz F., Peschel A.: Bacterial evasion of innate host defenses - the Staphylococcus aureus lesson. Int. J. Med. Microbiol. 2004, 294, 189-194.

29. Fitzgerald J. R.: The Staphylococcus intermedius group of bacterial pathogens: species reclassification, pathogenesis and the emergence of methicillin resistance. Vet. Dermatol. 2009, 20, 490-495.

30. Forsythe P. J., Hill P. B., Thoday K. L., Brown J.: Use of computerized image analysis to quantify staphylococcal adhesion to canine corneocytes: does breed and body site have any relevance to the pathogenesis of pyoderma? Vet. Dermatol. 2002, 13, 29-36.

31. Foster T. J.: Colonization and infection of the human host by staphylococci: adhesion, survival and immune evasion. Vet. Dermatol. 2009, 20, 456-470.

32. Futagawa-Saito K., Makino S., Sunaga F., Kato Y., Sakurai-Komada N. Ba-Thein W., Fukuyasu T.: Identification of first exfoliative toxin in Staphylococcus pseudintermedius. FEMS Microbiol. Lett. 2009, 301, 176-180.

33. Garthwaite G., Lloyd D. H., Thomsett L. R.: Location of immunoglobulins and complement (C3) at the surface and within the skin of dogs. J. Comp. Pathol. 1983, 93, 185-193.

34. Geoghegan J. A., Smith E. J., Speziale P., Foster T. J.: Staphylococcus pseudintermedius expresses surface proteins that closely resemble those from Staphylococcus aureus. Vet. Microbiol. 2009, 138, 345-352.

35. Griffeth G. C., Morris D. O., Abraham J. L., Shofer F. S., Rankin S. C. Screening for skin carriage of methicillin-resistant coagulase-positive staphylococci and Staphylococcus schleiferi in dogs with healthy and inflamed skin. Vet. Dermatol. 2008, 19, 142-149.

36. Hajjar A. M., O'Mahony D. S., Ozinsky A., Underhill D. M., Aderem A., Klebanoff S. J., Wilson C. B.: Cutting edge: functional interactions between toll-like receptor (TLR) 2 and TLR1 or TLR6 in response to phenol-soluble modulin. J. Immunol. 2001, 166, 15-19.

37. Hartmann F. A., White D. G., West S. E. H., Walker R. D., DeBoer D. J. Molecular characterization of Staphylococcus intermedius carriage by healthy dogs and comparison of antimicrobial susceptibility patterns to isolates from dogs with pyoderma. Vet. Microbiol. 2005, 108, 119-131.

38. Hashimoto M., Tawaratsumida K., Kariya H., Kiyohara A., Suda Y., Krikae F., Kirikae T., Götz F.: Not lipoteichoic acid but lipoproteins appear to be the dominant immunobiologically active compounds in Staphylococcus aureus. J. Immunol. 2006, 177, 3162-3169. 
39. Hendricks A., Schuberth H. J., Schueller K., Lloyd D. H.: Frequency of superantigen-producing Staphylococcus intermedius isolates from canine pyoderma and proliferation-inducing potential of superantigens in dogs. Res. Vet. Sci. 2002, 73, 273-277.

40. Hillier A., Lloyd D. H., Weese J. S., Blondeau J. M., Boothe D., Breitschwerdt E. Guardabassi L., Papich M. G., Rankin S., Turnidge J. D., Sykes J. E.: Guidelines for the diagnosis and antimicrobial therapy of canine superficial bacterial folliculitis (Antimicrobial Guidelines Working Group of the International Society for Companion Animal Infectious Diseases). Vet. Dermatol. 2014, 25, 163-e43.

41. Himsworth C. G., Patrick D. M., Parsons K., Feng A., Weese J. S.: Methicillinresistant Staphylococcus pseudintermedius in rats. Emerg. Infect. Dis. 2013, $19,169-170$

42. Hoffmann A. R., Patterson A. P., Diesel A., Lawhon S. D., Ly H. J., Elkins Stephenson C., Mansell J., Steiner J. M., Dowd S. E., Olivry T., Suchodolski $J$. S.: The skin microbiome in healthy and allergic dogs. PLoS One 2014, 9 , e83197.

43. Hoovels L. van, Vankeerberghen A., Boel A., van Vaerenbergh K., De Beenhouwer $H$.: First case of Staphylococcus pseudintermedius infection in a human. J. Clin. Microbiol. 2006, 44, 4609-4612.

44. Inden K., Kaneko J., Miyazato A., Yamamoto N., Mouri S., Shibuya Y., Nakamura K., Aoyagi T., Hatta M., Kunishima H., Hirakata Y., Itoh Y., Kaku M., Kawakami K.: Toll-like receptor 4-dependent activation of myeloid dendritic cells by leukocidin of Staphylococcus aureus. Microbes Infect. 2009, $11,245-253$.

45. Ihrke P. J.: An overview of bacterial skin disease in the dog. Br. Vet. J. 1987 $143,112-118$

46. Iyori K., Hisatsune J., Kawakami T., Shibata S., Murayama N., Ide K. Nagata M., Fukata T., Iwasaki T., Oshima K., Hattori M., Sugai M., Nishifuji K.: Identification of a novel Staphylococcus pseudintermedius exfoliative toxin gene and its prevalence in isolates from canines with pyoderma and healthy dogs. FEMS Microbiol. Lett. 2010, 312, 169-175.

47. Iyori K., Futagawa-Saito K., Hisatsune J., Yamamoto M., Sekiguchi M. Ide K., Son W. G., Olivry T., Sugai M., Fukuyasu T., Iwasaki T., Nishifuji K. Staphylococcus pseudintermedius exfoliative toxin EXI selectively digests canine desmoglein 1 and causes subcorneal clefts in canine epidermis. Vet. Dermatol. 2011, 22, 319-326.

48. Jaeger K., Linek M., Power H. T., Bettenay S. V., Zabel S., Rosychuk R. A. W., Mueller R. S.: Breed and site predispositions of dogs with atopic dermatitis: a comparison of five locations in three continents. Vet. Dermatol. 2010, 21, 118-122.

49. Keane K. A., Taylor D. J.: Slime-producing species in canine pyoderma. Vet. Rec. 1992, 130, 697-701.

50. Kizerwetter-Świda M., Chrobak-Chmiel D., Rzewuska M., Binek M.: Staphylococcus pseudintermedius - trudno rozpoznawalny patogen. Post. Mikrobiol. 2015, 54, 103-114

51. Koymans K. J., Feitsma L. J., Brondijk T. H., Aerts P. C., Lukkien E., Lössl P., van Kessel K. P., de Haas C. J., van Strijp J. A., Huizinga E. G.: Structura basis for inhibition of TLR2 by staphylococcal superantigen-like protein 3 (SSL3). Proc. Natl. Acad. Sci. USA 2015, 12, 11018-11023.

52. Kisich K. O., Howell M. D., Boguniewicz M., Heizer H. R., Watson N. U., Leung $D$. $Y$.: The constitutive capacity of human keratinocytes to kill Staphylococcus aureus is dependent on beta-defensin 3. J. Invest. Dermatol. 2007, 127, 2368 $-2380$

53. Kraus D., Peschel A.: Staphylococcus aureus evasion of innate antimicrobial defense. Future Microbiol. 2008, 3, 437-451.

54. Krishna S., Miller L. S.: Innate and adaptive immune responses against Staphylococcus aureus skin infections. Semin. Immunopathol. 2012, 34, 261-280.

55. Kumari S., Bonnet M. C., Ulvmar M. H., Wolk K., Karagianni N., Witte E., Uthoff-Hachenberg C., Renauld J. C., Kollias G., Toftgard R., Sabat R., Pasparakis M., Haase I.: Tumor necrosis factor receptor signaling in keratinocytes triggers interleukin-24-dependent psoriasis-like skin inflammation in mice. Immunity 2013, 39, 899-911.

56. Latronico F., Moodley A., Nielsen S. S., Guardabassi L.: Enhanced adherence of methicillin-resistant Staphylococcus pseudintermedius sequence type 71 to canine and human corneocytes. Vet. Res. 2014, 45, 70.

57. Leonard B. C., Marks S. L., Outerbridge C. A., Affolter V. K., Kananurak A., Young A., Moore P. F., Bannasch D. L., Bevins C. L.: Activity, expression and genetic variation of canine $\beta$-defensin 103: a multifunctional antimicrobial peptide in the skin of domestic dogs. J. Innate Immun. 2012, 4, 248-259.

58. Lloyd D. H., Garthwaite G.: Epidermal structure and surface topography of canine skin. Res. Vet. Sci. 1982, 33, 99-104.

59. Marsella R., Olivry T.: Animal models of atopic dermatitis. Clin. Dermatol. 2003, 21, 122-133.
60. Mason I. S., Lloyd D. H.: The role of allergy in the development of canine pyoderma. J. Small Anim. Pract. 1989, 30, 216-218.

61. Mason I. S., Lloyd D. H.: Factors influencing the penetration of bacterial antigens through canine skin. Von Tscarner C., Halliwell R.E.W. Advances in Veterinary Dermatology 1990, 1, 370-374.

62. Mason I. S., Lloyd D. H.: Scanning electron microscopical studies of the living epidermis and stratum corneum of dogs, [in:] Ihrke P. J., Mason I. S., White S. D.: Advances in Veterinary Dermatology 1993, 2, 131-139.

63. Mazzilli M., Zecconi A.: Assessment of epithelial cells' immune and inflammatory response to Staphylococcus aureus when exposed to a macrolide. J. Dairy Res. 2010, 77, 404-410.

64. McEwan N. A.: Adherence by Staphylococcus intermedius to canine keratinocytes in atopic dermatitis. Res. Vet. Sci. 2000, 68, 279-283.

65. McEwan N. A., Kalna G., Mellor D.: A comparison of adherence by four strains of Staphylococcus intermedius and Staphylococcus hominis to canine corneocytes collected from normal dogs and dogs suffering from atopic dermatitis. Res. Vet. Sci. 2005, 78, 193-198.

66. McEwan N. A., Mellor D., Kalna G.: Adherence by Staphylococcus intermedius to canine corneocytes: a preliminary study comparing noninflamed and inflamed atopic canine skin. Vet. Dermatol. 2006, 17, 151-154.

67. Miller L. S.: Toll-like receptors in skin. Adv. Dermatol. 2008, 24, 71-87.

68. Miszczak D., Stońska A., Golke A., Cymerys J.: Herpesviruses survival strategies - latency and apoptosis. Postepy Hig. Med. Dosw. (Online) 2013, 67, 276-287.

69. Momota Y., Shimada K., Noguchi A., Saito A., Nozawa S., Niina A., Tani K., Azakami D., Ishiota K., Sako T.: The modified corneocyte surface area measurement as an index of epidermal barrier properties: inverse correlation with transepidermal water loss. Vet. Dermatol. 2016, 27, 67-e19.

70. Moodley A., Stegger M., Zakour N. L., Fitzgerald J. R., Guardabassi L. Tandem repeat sequence analysis of staphylococcal protein A (spa) gene in methicillin resistant Staphylococcus pseudintermedius. Vet. Microbiol. 2009, 135, 320-326.

71. Morris D. O., Rook K. A., Shofer F. S., Rankin S. C.: Screening of Staphylococcus aureus, Staphylococcus intermedius and Staphylococcus schleiferi isolates obtained from small companion animals for antimicrobial resistance: a retrospective review of 749 isolates (2003-04). Vet. Dermatol. 2006, 17, 332-337.

72. Muller G.: Bacterial skin diseases, [in:] Scott D. M. W., Griffin C. (eds.): Muller and Kirk's Small Animal Dermatology. $6^{\text {th }}$ edn. W.B. Saunders Co Philadelphia, PA 2000, p. 274-335

73. Mullin J., Carter S., Williams N., McEwan N., Nuttall T.: Transcription of canine toll-like receptor $2, \beta$-defensin 1 and $\beta$-defensin 103 in infected atopic skin, non-infected atopic skin, healthy skin and the CPEK cell line. Vet. Microbiol. 2013, 162, 700-706.

74. Müller-Anstett M. A., Muller P., Albrecht T., Nega M., Wagener J., Gao Q., Kaesler S., Schaller M., Biedermann T., Götz F.: Staphylococcal peptidoglycan co-localizes with Nod 2 and TLR2 and activates innate immune response via both receptors in primary murine keratinocytes. PLoS One 2010, 5, e13153.

75. Onogawa T.: Staphylococcal alpha-toxin synergistically enhances inflammation caused by bacterial components. FEMS Immunol. Med. Microbiol. 2002 $33,15-21$.

76. Ouhara K., Komatsuzawa H., Kawai T., Nishi H., Fujiwara T., Fujiue Y., Kuwabara M., Sayama K., Hashimoto K., Sugai M.: Increased resistance to cationic antimicrobial peptide LL-37 in methicillin-resistant strains of Staphylococcus aureus. J. Antimicrob. Chemother. 2008, 61, 1266-1269.

77. Parker D., Prince A.: Staphylococcus aureus induces type I IFN signaling in dendritic cells via TLR9. J. Immunol. 2012, 189, 4040-4046.

78. Paul N. C., Latronico F., Moodley A., Nielsen S. S., Damborg P., Guardabassi L.: In vitro adherence of Staphylococcus pseudintermedius to canine corneocytes is influenced by colonization status of corneocyte donors. Vet. Res. 2013, 44, 52 .

79. Pietrocola G., Gianotti V., Richards A., Nobile G., Geoghegan J. A., Rindi S., Monk I. R., Bordt A. S., Foster T. J., Fitzgerald J. R., Speziale P.: Fibronectin binding proteins SpsD and SpsL both support invasion of canine epithelial cells by Staphylococcus pseudintermedius. Infect. Immun. 2015, 83, 4093-4102.

80. Prévost G., Bouakham T., Piemont Y., Monteil H.: Characterisation of a synergohymenotropic toxin produced by Staphylococcus intermedius. FEBS Lett. 1995, 376, 135-140.

81. Proft T., Fraser J. D.: Bacterial superantigens. Clin. Exp. Immunol. 2003, 133, 299-306.

82. Robinson J.: Colonization and infection of the respiratory tract: What do we know? Pediatr. Child. Health 2004, 9, 21-24.

83. Rooijakkers S. H., Ruyken M., Roos A., Daha M. R., Presanis J. S., Sim R. B., van Wamel W. J., van Kessel K. P., van Strijp J. A.: Immune evasion by a staphylococcal complement inhibitor that acts on $\mathrm{C} 3$ convertases. Nat. Immunol. 2005, 6, 920-927. 
84. Rubin J. E., Chirino-Trejo M.: Prevalence, sites of colonization, and antimicrobial resistance among Staphylococcus pseudintermedius isolated from healthy dogs in Saskatoon, Canada. J. Vet. Diagn. Invest. 2011, 23, 351-354.

85. Ruscher C., Lübke-Becker A., Semmler J., Wleklinski C. G., Paasch A., Soba A., Stamm I., Kopp P., Wieler L. H., Walther B.: Widespread rapid emergence of a distinct methicillin- and multidrug-resistant Staphylococcus pseudintermedius (MRSP) genetic lineage in Europe. Vet. Microbiol. 2010, 144, 340-346.

86. Ruzauskas M., Couto N., Pavilonis A., Klimiene I., Siugzdiniene R., Virgailis M., Vaskeviciute L., Anskiene L., Pomba C.: Characterization of Staphylococcus pseudintermedius isolated from diseased dogs in Lithuania. Pol. J. Vet. Sci. 2016, 19, 7-14.

87. Saijonmaa-Koulumies L. E., Lloyd D. H.: Adherence of Staphylococcus intermedius to canine corneocytes in vitro. Vet. Dermatol. 2002, 13, 169-176.

88. Sakoulas G., Guram K., Reyes K., Nizet V., Zervos M.: Human cathelicidin LL-37 resistance and increased daptomycin MIC in methicillin-resistant Staphylococcus aureus strain USA 600 (ST45) are associated with increased mortality in a hospital setting. J. Clin. Microbiol. 2014, 52, 2172-2174.

89. Santoro D., Bunick D., Graves T. K., Segre M.: Evaluation of canine antimicrobial peptides in infected and noninfected chronic atopic skin. Vet. Dermatol 2013, 24, 39-47.

90. Santoro D., Maddox C. W.: Canine antimicrobial peptides are effective against resistant bacteria and yeasts. Vet. Dermatol. 2014, 25, 35-39.

91. Schmidt V., Nuttal T., Fazakerley J., McEwan N.: Staphylococcus intermedius binding to immobilized fibrinogen, fibronectin and cytokeratin in vitro. Vet. Dermatol. 2009, 20, 501-508.

92. Schmidt V., Williams N. J., Pinchbeck G., Corless C. E., Shaw S., McEwan N., Dawson S., Nuttall T.: Antimicrobial resistance and characterization of staphylococci isolated from healthy Labrador retrievers in the United Kingdom. BMC Vet. Res. 2014, 10, 17.

93. Schwarz S., Kadlec K., Strommenger B.: Methicillin-resistant Staphylococcus aureus and Staphylococcus pseudintermedius detected in the BfT-Germ Vet monitoring programme 2004-2006 in Germany. J. Antimicrob. Chemother. 2008, 61, 282-285

94. Scott D. W., Muller W. H., Griffin C. E.: Skin immune system and allergic skin diseases, [in:] Muller and Kirk's small animal dermatology. W. B. Saunders Philadelphia 2001, p. 543-666.

95. Sieprawska-Lupa M., Mydel P., Krawczyk K., Wojcik K., Puklo M., Lupa B., Suder P., Silberring J., Reed M., Pohl J., Shafer W., McAleese F., Foster T., Travis J., Potempa J.: Degradation of human antimicrobial peptide LL-37 by Staphylococcus aureus-derived proteinases. Antimicrob. Agents Chemother. 2004, 48, 4673-4679.

96. Simou C., Thoday K. L., Forsythe P. J., Hill P. B.: Adherence of Staphylococcus intermedius to corneocytes of healthy and atopic dogs: effect of pyoderma, pruritus score, treatment and gender. Vet. Dermatol. 2005a, 16, 385-391.

97. Simou C., Hill P. B., Forsythe P. J., Thoday K. L.: Species specificity in the adherence of staphylococci to canine and human corneocytes: a preliminary study. Vet. Dermatol. 2005b, 16, 156-161.

98. Sorensen O. E., Follin P., Johnsen A. H., Calafat J., Tjabringa G. S., Hiemstra P. S., Borregaard N.: Human cathelicidin, hCAP-18, is processed to the antimicrobial peptide LL-37 by extracellular cleavage with proteinase 3 . Blood 2001, 97, 3951-3959.

99. Stappers M. H., Thys Y., Oosting M., Plantinga T. S., Ioana M., Reimnitz P., Mouton J. W., Netea M. G., Joosten L. A., Gyssens I. C.: TLR1, TLR2, and
TLR6 gene polymorphisms are associated with increased susceptibility to complicated skin and skin structure infections. J. Infect. Dis. 2014, 210, 311 -318 .

100.Summers J. F., Brodbelt D. C., Forsythe P. J., Loeffler A., Hendricks A.: The effectiveness of systemic antimicrobial treatment in canine superficial and deep pyoderma: a systemic review. Vet. Dermatol. 2012, 23, 305-e61.

101.Takeda K., Takeuchi O., Akira S.: Recognition of lipopeptides by Toll-like receptors. J. Endotoxin Res. 2002, 8, 459-463.

102.Tanabe T., Toyoguchi M., Hirano F., Chiba M., Onuma K., Sato H.: Prevalence of staphylococcal enterotoxins in Staphylococcus pseudintermedius isolates from dogs with pyoderma and healthy dogs. Microbiol. Immunol. 2013, 57, 651-654.

103.Taylor J. M., Heinrichs D. E.: Transferrin binding in Staphylococcus aureus: involvement of a cell wall-anchored protein. Mol. Microbiol. 2002, 43, 1603 $-1614$.

104.Terauchi R., Sato H., Hasegawa T., Yamaguchi T., Aizawa C., Maehara N.: Isolation of exfoliative toxin from Staphylococcus intermedius and its local toxicity in dogs. Vet. Microbiol. 2003, 94, 19-29.

105.Terui T., Kato T., Tagami H.: Stratum corneum activation of complement through the antibody-independent alternative pathway. J. Investig. Dermatol. 1989, 92, 593-597.

106.Travassos L. H., Girardin S. E., Philpott D. J., Blanot D., Nahori M. A. Werts C., Boneca I. G.: Toll-like receptor 2-dependent bacterial sensing does not occur via peptidoglycan recognition. EMBO Rep. 2004, 10, 1000-1006.

107.Walev I., Weller U., Strauch S., Foster T., Bhakdi S.: Selective killing of human monocytes and cytokine release provoked by sphingomyelinase (beta-toxin) of Staphylococcus aureus. Infect. Immun. 1996, 64, 2974-2979.

108.Wang G. S., Epand R. F., Mishra B., Lushnikova T., Thomas V. C., Bayles $K$. W., Epand R. M.: Decoding the functional roles of cationic side chains of the major antimicrobial region of human cathelicidin LL-37. Antimicrob. Agents Chemother. 2012, 56, 845-856.

109. Watanabe I., Ichiki M., Shiratsuchi A., Nakanishi Y.: TLR2-mediated survival of Staphylococcus aureus in macrophages: a novel bacterial strategy against host innate immunity. J. Immunol. 2007, 178, 4917-4925.

110. Weese J. S., van Duijkeren E.: Methicillin-resistant Staphylococcus aureus and Staphylococcus pseudintermedius in veterinary medicine. Vet. Microbiol. 2010, 140, 418-429.

111. Wettstein K., Descloux S., Rossano A., Perreten V.: Emergence of methicillin-resistant Staphylococcus pseudintermedius in Switzerland: three cases of urinary tract infections in cats. Schweiz Arch. Tierheilkd. 2008, 150, 339-343.

112. Yokoyama R., Itoh S., Kamoshida G., Takii T., Fujii S., Tsuji T., Onozaki K.: Staphylococcal superantigen-like protein 3 binds to the Toll-like receptor 2 extracellular domain and inhibits cytokine production induced by Staphylococcus aureus, cell wall component, or lipopeptides in murine macrophages. Infect. Immun. 2012, 80, 2816-2825.

113. Yoon J. W., Lee G. J., Lee S. Y., Park C., Yoo J. H., Park H. M.: Prevalence of genes for enterotoxins, toxic shock syndrome toxin 1 and exfoliative toxin among clinical isolates of Staphylococcus pseudintermedius from canine origin. Vet. Dermatol. 2010, 21, 484-489.

Corresponding author: Anna Golke PhD, Ciszewskiego 8, 02-786 Warsaw; e-mail: anna_golke@sggw.pl 